\title{
Optimum Power Control for Transmitter Cooperation in OFDMA Based Wireless Networks
}

\author{
Sezi Bakım and Onur Kaya \\ Department of Electrical and Electronics Engineering \\ Işık University, Şile, Istanbul, Turkey \\ sezi.bakim@isik.edu.tr_onurkaya@isikun.edu.tr
}

\begin{abstract}
For a cooperative orthogonal frequency division multiple access (OFDMA) system with two transmitters (TXs), and full channel state information (CSI), we obtain the optimal power allocation (PA) policies which maximize the rate region achievable by a recently introduced version of block Markov superposition encoding (BMSE): inter-subchannel cooperative encoding (ISCE) [1]. We provide the optimality conditions that need to be satisfied by the powers associated with the transmitted codewords. We propose two algorithms that yield the optimal power distribution: a subgradient algorithm which achieves an arbitrary rate point on the achievable rate region boundary, and an iterative waterfillinglike algorithm which maximizes the sum rate, and converges much faster. We observe that, utilization of power control to take advantage of the diversity offered by the cooperative OFDMA system, not only leads to a remarkable improvement in achievable rates, but also may help determine how the subchannels have to be instantaneously allocated to various tasks in cooperation.
\end{abstract}

\section{INTRODUCTION}

The ability of OFDMA to cope with both intersymbol and interuser interference, combined with its low complexity of implementation have made it a popular choice for the next generation wireless networks. As a result, the problem of resource allocation in OFDMA systems was studied extensively in the literature. One example is [2], where it was proved that in an OFDMA uplink system, allocating subcarriers to the users with the maximum marginal rate is a necessary condition for maximizing the system throughput. A similar problem was solved in [3] using KKT conditions, by optimizing a utility function which was assumed to be a function of the rates. In [4], a low-complexity algorithm for subcarrier, power, and rate allocation for OFDMA was proposed, to maximize the sum rate under individual rate constraints to guarantee fairness. These works, as well as many others on OFDMA, naturally assume orthogonal multiple access, thereby choosing to avoid interference. However, like all orthogonal transmission techniques, OFDMA incurs some rate penalty, caused by the orthogonalization. Moreover, in wireless channels, "interference" is in fact free side information, and gives rise to the concept of user cooperation, if taken advantage of by the system design. Hence, in this paper, we focus on resource allocation for a cooperative OFDMA model, which allows subchannels to be shared by pairs of transmitters, thereby treating interference as information, and allowing mutual cooperation among the transmitters over each subchannel.

Besides its natural use in the uplink within each cell, the cooperative OFDMA system analyzed in this paper is especially useful in multicell cooperation scenarios, some examples of which are illustrated in Figure 1:

- In the downlink scenario shown in Figure 1(a), a cell edge mobile station (MS) acts as a receiver (RX), and two

This work was supported by TUBITAK Grant 108E208.

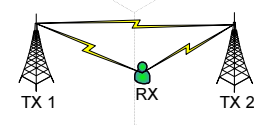

(a) Downlink cooperation scenario.

(b) Uplink cooperation scenario.

Fig. 1. Multicell cooperation scenarios, based on cooperative OFDMA.

base stations (BSs) act as transmitters (TX 1 and TX 2), which creates a two-transmitter multiple access channel (MAC). Assuming that the MS is already likely to receive data from both BSs in a soft handoff scenario, we can purposely assign the same subchannels to both BSs, so that the MS simultaneously receives data from both base stations, and jointly decodes it. Meanwhile the BSs, which potentially have reliable line of sight links among each other, can cooperate on each subchannel so that they can obtain coherent combining gain at the BS. This approach also allows multiple independent data streams arriving at separate BSs to be cooperatively routed to the MS.

- In the uplink scenario shown in Figure 1(b), two cell edge MSs (TX 1 and TX 2), each of which belong to one of two adjacent cells, try to communicate with one of the BSs: either RX a or RX b. The resulting model for each BS is once again a two-transmitter MAC. Since the two MSs are located closely, assigning the same subchannels to them will create high quality side information, and the MSs can cooperatively transmit to the BS of their choice to maximize their rates, rather than sticking with their own BS. More interestingly, assigning the same subchannels to both MSs allows for frequency (subchannel) reuse in two adjacent cells, thereby increasing user capacity.

The overheard information in a typical wireless MAC, is captured by modeling the system as a MAC with generalized feedback (MAC-GF) [5]. In [5], achievable rates for the MACGF were obtained based on BMSE and backward decoding. In [6], these encoding and decoding techniques were applied to a Gaussian MAC in fading, and the resulting rate regions were characterized. In [7], PA policies that maximize the rates achievable by BMSE for the same model were obtained.

While the above works all deal with a scalar MAC-GF, some works on resource allocation for user cooperation in vector channels, specifically OFDMA, also exist. A cooperative OFDMA system where each user is allowed to transmit and receive at the same time, but necessarily on different subcarriers, was considered in [8]. Subcarrier and power allocation schemes for a time-division duplex amplify and forward protocol were employed in [9] with the aim of maximizing system 
throughput and enhancing fairness in a cooperative OFDMA uplink system. Resource allocation and cooperative partner selection in cooperative OFDM networks was investigated with the objective of minimizing the overall power in [10]. However, these works consider either a one sided cooperation strategy, or a mutually cooperative strategy based on two parallel dedicated relay channels, or mutual cooperation based on a time division protocol. A more general cooperative OFDMA model based on parallel MAC-GFs, which does not make any prior assumptions about the way in which the subchannels are assigned to the TXs, was introduced in [1], but there, PA as a function of fading states, which can further take advantage of the temporal diversity over each subchannel, was not considered.

In this paper, we obtain the optimum PA policies that maximize the rate region achievable by the full-duplex ISCE strategy of [1]. Despite the complex re-encoding structure employed in ISCE, and the fact that the powers allocated to each subchannel have to satisfy a sum power constraint over subchannels, the achievable rate region turns out to be of a relatively similar form to its scalar counterpart, and we are able to extend some properties of the optimal PA derived in [7] for scalar cooperative MAC, to cooperative OFDMA. As a result, the weighted sum of rates, which can be used to obtain any point on the rate region boundary, becomes concave, and convex optimization techniques can be employed. For the general case of maximization of an arbitrary rate point, we employ a projected subgradient algorithm that converges to the optimum. For the special case of sum rate maximization, we derive the optimality conditions, and closed form expressions for optimum powers analytically. We then propose an efficient iterative algorithm with a much lower complexity, that obtains the sum-rate-optimal powers. As a result, we demonstrate that by jointly exploiting the diversity provided by OFDMA's parallel subchannels, and the temporal diversity created by the time varying channel, we obtain very promising gains in achievable rates. More interestingly, we observe that the optimal PA may automatically dictate that some subchannels are assigned exclusively to certain TXs/tasks, depending on the instantaneous channel state.

\section{System Model}

We consider a full-duplex cooperative OFDMA system with two TXs and one RX, operating over $N$ subchannels (see Fig. 2), modelled by,

$$
\begin{aligned}
& Y_{0}^{(i)}=h_{10}^{(i)} X_{1}^{(i)}+h_{20}^{(i)} X_{2}^{(i)}+Z_{0}^{(i)}, \\
& Y_{1}^{(i)}=h_{21}^{(i)} X_{2}^{(i)}+Z_{1}^{(i)}, \\
& Y_{2}^{(i)}=h_{12}^{(i)} X_{1}^{(i)}+Z_{2}^{(i)},
\end{aligned}
$$

where, for each subchannel $i \in\{1, \ldots, N\}, X_{k}^{(i)}$ is the symbol transmitted by node $k, Z_{l}^{(i)}$ is the zero-mean additive white Gaussian noise at node $l, h_{k l}^{(i)}$ is the fading coefficient between nodes $k$ and $l$, and $Y_{l}^{(i)}$ is the symbol received at node $l$; with $k \in\{1,2\}, l \in\{0,1,2\}$ and $k \neq l$. Here, the $\mathrm{RX}$ is denoted by $l=0$. The variance of $Z_{l}^{(i)}$ is given by $\sigma_{l}^{(i)^{2}}$. To simplify the notation throughout the paper, we define the normalized powerfading coefficients $s_{k l}^{(i)}=\frac{\left(h_{k l}^{(i)}\right)^{2}}{\sigma_{l}^{(i)^{2}}}$, and the Gaussian capacity

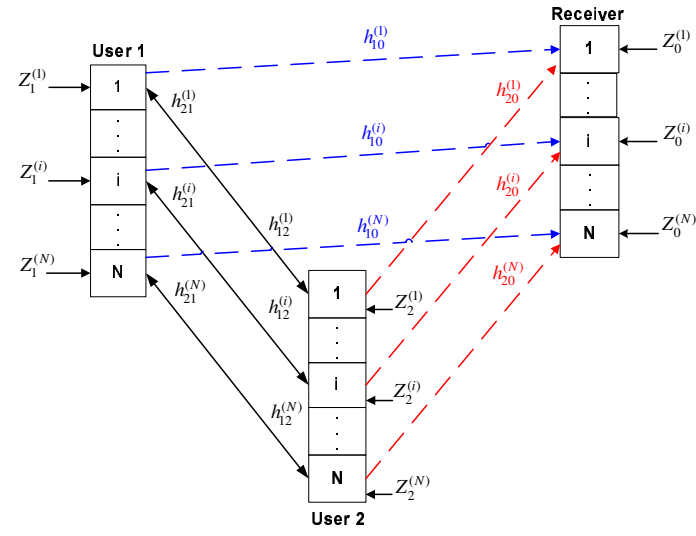

Fig. 2. Gaussian cooperative OFDMA channel.

function $C(x) \triangleq \frac{1}{2} \log (1+x)$.

We assume that the TXs and the RX have full CSI of both the cooperative links and the direct link. Therefore, the TXs can adapt their transmitted symbols $X_{k}^{(i)}$ as a function of the joint fading state $\mathbf{s}$. Note that there are two ways to view the channel adaptive transmission that maximizes the long term (ergodic) achievable rates: we can either use a variable power variable rate codebook, as in [11], or we can use a single codebook, whose rate is supported by the channel in the long term, and perform the channel adaptation by simply multiplying entries from this codebook by channel adaptive powers, as in [12]. In this paper, we employ the latter approach.

\section{Achievable Rates for Cooperative OFDMA}

To establish the cooperation among the TXs, we employ a channel adaptive version of the ISCE proposed in [1]. In ISCE, the message of each TX $k \in\{1,2\}$ is divided into two submessages, $w_{k 0}$ and $w_{k j}$, intended to be decoded at the RX and cooperative partner $j \in\{1,2\}$ respectively, which are further divided into $N$ submessages each,

$$
w_{k 0}=\left\{w_{k 0}^{(1)}, \ldots, w_{k 0}^{(N)}\right\}, \quad w_{k j}=\left\{w_{k j}^{(1)}, \ldots, w_{k j}^{(N)}\right\},
$$

with the respective rates $\left\{R_{k 0}^{(1)}, \ldots, R_{k 0}^{(N)}\right\}$ and $\left\{R_{k j}^{(1)}, \ldots, R_{k j}^{(N)}\right\}$, to be transmitted over disjoint subchannels. is established at the cooperating partner, the cooperative messages are repartitioned, to be transmitted to the destination in the next block, over potentially different subchannels, and at different rates, i.e.,

$$
w_{12}=\left\{v_{12}^{(1)}, \ldots, v_{12}^{(N)}\right\}, \quad w_{21}=\left\{v_{21}^{(1)}, \ldots, v_{21}^{(N)}\right\},
$$

with the respective rates $\left\{R_{12}^{\prime(1)}, \ldots, R_{12}^{\prime(N)}\right\},\left\{R_{21}^{\prime(1)}, \ldots, R_{21}^{\prime(N)}\right\}$. Then, the encoding in block $b \in\{1, \ldots, B\}$ is performed by assigning codewords $X_{k 0}^{(i)}, X_{k j}^{(i)}, U_{k}^{(i)}, i=1, \ldots, N$, selected from codebooks randomly generated according to unit Gaussian distributions, to messages from the previous and current blocks (and their estimates, denoted by $\hat{v}_{j k}^{(i)}$ ),

$$
\begin{aligned}
& X_{k 0}^{(i)}\left(w_{k 0}^{(i)}[b],\left(v_{k j}^{(i)}[b-1], \hat{v}_{j k}^{(i)}[b-1]\right)\right), \\
& X_{k j}^{(i)}\left(w_{k j}^{(i)}[b],\left(v_{k j}^{(i)}[b-1], \hat{v}_{j k}^{(i)}[b-1]\right)\right), \\
& U_{k}^{(i)}\left(v_{k j}^{(i)}[b-1], \hat{v}_{j k}^{(i)}[b-1]\right),
\end{aligned}
$$


and superposing them to obtain the overall codeword of each $\mathrm{TX}$. The decoding at the RX is performed using backwards decoding [5].

Note that, [1] does not utilize instantaneous CSI to adapt the instantaneous transmission powers. In order to further take advantage of the diversity provided by the OFDMA system, in this paper, we propose a channel adaptive version of the encoding strategy in [1], where we scale each of the above codewords by variable powers,

$$
X_{k}^{(i)}=\sqrt{p_{k 0}^{(i)}(\mathbf{s})} X_{k 0}^{(i)}+\sqrt{p_{k j}^{(i)}(\mathbf{s})} X_{k j}^{(i)}+\sqrt{p_{U_{k}}^{(i)}(\mathbf{s})} U_{k}^{(i)},
$$

where $k, j \in\{1,2\}, k \neq j, i=1, \cdots, N$, and the powers are subject to the average constraints,

$\sum_{i} E\left[p_{k 0}^{(i)}(\mathbf{s})+p_{k j}^{(i)}(\mathbf{s})+p_{U_{k}}^{(i)}(\mathbf{s})\right] \triangleq \sum_{i} E\left[p_{k}^{(i)}(\mathbf{s})\right] \leq \bar{p}_{k}$.

With this adaptive encoding strategy, we can extend [1, Corollary 2] to parallel Gaussian channels, to yield the achievable rate region for power controlled ISCE: the achievable rate region is given by the closure of the convex hull of all rate pairs $\left(R_{1}, R_{2}\right)$ satisfying

$$
\begin{aligned}
& R_{1}<\sum_{i}^{N} E\left[C\left(\frac{s_{12}^{(i)} p_{12}^{(i)}(\mathbf{s})}{s_{12}^{(i)} p_{10}^{(i)}(\mathbf{s})+1}\right)+C\left(s_{10}^{(i)} p_{10}^{(i)}(\mathbf{s})\right)\right] \\
& R_{2}<\sum_{i}^{N} E\left[C\left(\frac{s_{21}^{(i)} p_{21}^{(i)}(\mathbf{s})}{s_{21}^{(i)} p_{20}^{(i)}(\mathbf{s})+1}\right)+C\left(s_{20}^{(i)} p_{20}^{(i)}(\mathbf{s})\right)\right] \\
& R_{1}+R_{2}<\min \left\{\sum _ { i } ^ { N } E \left[C \left(s_{10}^{(i)} p_{1}^{(i)}(\mathbf{s})+s_{20}^{(i)} p_{2}^{(i)}(\mathbf{s})\right.\right.\right. \\
& \left.\left.+2 \sqrt{s_{10}^{(i)} s_{20}^{(i)} p_{U_{1}}^{(i)}(\mathbf{s}) p_{U_{2}}^{(i)}(\mathbf{s})}\right)\right], \sum_{i}^{N} E\left[C\left(\frac{s_{12}^{(i)} p_{12}^{(i)}(\mathbf{s})}{s_{12}^{(i)} p_{10}^{(i)}(\mathbf{s})+1}\right)\right. \\
& \left.\left.+C\left(\frac{s_{21}^{(i)} p_{21}^{(i)}(\mathbf{s})}{s_{21}^{(i)} p_{20}^{(i)}(\mathbf{s})+1}\right)+C\left(s_{10}^{(i)} p_{10}^{(i)}(\mathbf{s})+s_{20}^{(i)} p_{20}^{(i)}(\mathbf{s})\right)\right]\right\}
\end{aligned}
$$

where the convex hull is taken over all valid PA policies.

\section{Channel Adaptive Power Allocation}

If we set $N=1$ in (11)-(13), the problem reduces to a scalar cooperative MAC. In [7], it was shown for this case that, based on the instantaneous channel state, the optimal PA dictates that each TX either sends cooperative information, or fresh information, but not both. Although in OFDMA, there is a sum power constraint over the subchannels, and one would expect the PA over each subchannel to be dependent on the powers assigned to the other subchannels, we show that many properties of the optimal PA for OFDMA remain surprisingly parallel to those in the scalar case [7], and the codewords that should be used over each subchannel are determined solely by the instantaneous fading coefficients over that particular subchannel, as stated in the following theorem:

Theorem 1: The PA policy that maximizes the sum rate of a cooperative OFDMA system using ISCE should satisfy;

1) $p_{10}^{(i) *}(\mathbf{s})=p_{20}^{(i) *}(\mathbf{s})=0$, if $s_{12}^{(i)}>s_{10}^{(i)}$ and $s_{21}^{(i)}>s_{20}^{(i)}$

2) $p_{10}^{(i) *}(\mathbf{s})=p_{21}^{(i) *}(\mathbf{s})=0$, if $s_{12}^{(i)}>s_{10}^{(i)}$ and $s_{21}^{(i)} \leq s_{20}^{(i)}$

3) $p_{12}^{(i) *}(\mathbf{s})=p_{20}^{(i) *}(\mathbf{s})=0$, if $s_{12}^{(i)} \leq s_{10}^{(i)}$ and $s_{21}^{(i)}>s_{20}^{(i)}$
4) $p_{12}^{(i) *}(\mathbf{s})=p_{21}^{(i) *}(\mathbf{s})=0$ or $p_{10}^{(i) *}(\mathbf{s})=p_{21}^{(i) *}(\mathbf{s})=0$ or $p_{12}^{(i) *}(\mathbf{s})=p_{20}^{(i) *}(\mathbf{s})=0$, if $s_{12}^{(i)} \leq s_{10}^{(i)}$ and $s_{21}^{(i)} \leq s_{20}^{(i)}$.

Proof: Let us assume that we know the total optimal power $p_{k}^{(i) *}(\mathbf{s})$, allocated to each subchannel $i$ at each channel state s. For ISCE, the sum rate (13) is maximized if each argument of the minimum operation is maximized. The first argument is insensitive to the choice of $p_{k 0}^{(i) *}(\mathbf{s})$ or $p_{k j}^{(i) *}(\mathbf{s})$, as long as their sum is fixed; whereas the second argument is maximized if we separately maximize its summands for each $i$. The result follows by noting that this is equivalent to $N$ independent optimization problems, each yielding a scalar case, and [7, Proposition 1] holds, giving the desired result.

An important observation is that, setting two of the powers equal to zero as suggested by Theorem 1, is also optimal for the entire rate region maximization, as the right hand sides of all three constraints are maximized by choosing the powers according to Theorem $1 .{ }^{1}$ Therefore, from now on we focus only on policies that satisfy Theorem 1 . But then, it is easy to check that the rate constraints in (11)-(13) now become concave in the power vector $\mathbf{p}(\mathbf{s})=\left[p_{10}^{(i) *}(\mathbf{s}), p_{12}^{(i) *}(\mathbf{s}), p_{U_{1}}^{(i) *}(\mathbf{s}), p_{20}^{(i) *}(\mathbf{s})\right.$, $\left.p_{21}^{(i) *}(\mathbf{s}), p_{U_{2}}^{(i) *}(\mathbf{s}), i=1, \ldots, N\right]$, lending themselves to well known techniques in convex optimization, which we discuss in the next sections.

\section{A. Maximization of the Weighted Sum of Rates}

Since all bounds of the achievable rate region are concave in powers, so is any weighted sum $\mu_{1} R_{1}+\mu_{2} R_{2}$ at the corners. Moreover, it is easy to show that the rate region is strictly conxex [7], [12]. Therefore, we can obtain points on the rate region boundary by maximizing $R_{\mu}=\mu_{1} R_{1}+\mu_{2} R_{2}$. Assuming $\mu_{1}>\mu_{2}$ without loss of generality, the optimization problem can be stated as:

$$
\begin{aligned}
\max _{\mathbf{p}(\mathbf{s})} & \left(\left(\mu_{1}-\mu_{2}\right) \sum_{i} R_{1}^{(i)}+\mu_{2} \sum_{i}\left(R_{1}^{(i)}+R_{2}^{(i)}\right)\right) \\
\text { s.t. } & \sum_{i} E\left[p_{k 0}^{(i)}(\mathbf{s})+p_{k j}^{(i)}(\mathbf{s})+p_{U_{k}}^{(i)}(\mathbf{s})\right] \leq \bar{p}_{k} \\
& p_{k 0}^{(i)}(\mathbf{s}), p_{k j}^{(i)}(\mathbf{s}), p_{U_{k}}^{(i)}(\mathbf{s}) \geq 0, k, j \in\{1,2\}, k \neq j
\end{aligned}
$$

where $\left\{R_{1}, R_{2}\right\}$ is the corner of the pentagon obtained from (11)-(13) for a given PA policy. Unfortunately, due to the minimum operation in (13), the gradient of the objective function does not exist everywhere. In particular, there are two gradient vectors, depending on which argument of the minimum in (13) is active. Yet, these vectors may be viewed instead as subgradients, which makes it possible to employ the method of projected subgradient, for power optimization. Due to the convex nature of our problem, this method is guaranteed to converge to the global optimum, provided a diminishing stepsize normalized by the norm of the subgradient is used [13]. Since the calculation of the subgradients requires rather tedious formulas which give little insight, we will instead directly provide the achievable rate region, and some samples from the resulting power allocation policy in Section V.

\footnotetext{
${ }^{1}$ We choose the first option in case 4, which may cause a slight deviation from optimality for the sum rate. However, the probability of case 4 occuring is very low practical cooperative settings, and this suboptimality can be ignored, as it has been done in [7].
} 
The major drawbacks of the subgradient algorithm are its slow rate of convergence, and complexity. As the number of subchannels increase, so does the size of the vector of power variables, making the process of computing the subgradients, and the projection operations formidable. In the next section, we propose an alternative approach, where we obtain the analytical expressions for sum-rate-optimal powers, and develop an iterative algorithm which converges much faster. This approach can also be extended to arbitrary rate point maximization, and will be discussed in a journal version.

\section{B. Sum Rate Maximization}

To simplify the exposition, we assume that we always operate in case 1 , where $s_{12}^{(i)}>s_{10}^{(i)}$ and $s_{21}^{(i)}>s_{20}^{(i)}$, yielding $p_{10}^{(i)}(\mathbf{s})=0$ and $p_{20}^{(i)}(\mathbf{s})=0$. The sum rate maximization problem can then be stated as

$$
\begin{aligned}
\max _{\mathbf{p}(\mathbf{s})} & R_{s} \\
R_{s} & \leq \sum_{i} E\left[C\left(p_{12}^{(i)}(\mathbf{s}) s_{12}^{(i)}\right)+C\left(p_{21}^{(i)}(\mathbf{s}) s_{21}^{(i)}\right)\right] \\
R_{s} & \leq \sum_{i} E\left[\frac{1}{2} \log \left(A^{(i)}\right)\right] \\
& \sum_{i}\left(E\left[p_{12}^{(i)}(\mathbf{s})\right]+E\left[p_{U_{1}}^{(i)}(\mathbf{s})\right]\right) \leq \bar{p}_{1} \\
& \sum_{i}\left(E\left[p_{21}^{(i)}(\mathbf{s})\right]+E\left[p_{U_{2}}^{(i)}(\mathbf{s})\right]\right) \leq \bar{p}_{2} \\
& p_{12}^{(i)}(\mathbf{s}), p_{U_{1}}^{(i)}(\mathbf{s}), p_{21}^{(i)}(\mathbf{s}), p_{U_{2}}^{(i)}(\mathbf{s}) \geq 0, \quad \forall \mathbf{s}
\end{aligned}
$$

where $A^{(i)}$ is defined as

$$
A^{(i)}=1+s_{10}^{(i)} p_{1}^{(i)}(\mathbf{s})+s_{20}^{(i)} p_{2}^{(i)}(\mathbf{s})+2 \sqrt{s_{10}^{(i)} s_{20}^{(i)} p_{U_{1}}^{(i)}(\mathbf{s}) p_{U_{2}}^{(i)}(\mathbf{s})} \text {. }
$$

Assigning Lagrange multipliers $\gamma_{1}, \gamma_{2}, \lambda_{1}, \lambda_{2}$ to constraints (15)-(18), and $\epsilon_{t}^{(i)}(\mathbf{s}), t=1,2,3,4$, to the positivity constraints (19), the KKT conditions for optimality can be obtained as,

$$
\begin{gathered}
\gamma_{1} \frac{s_{k j}^{(i)}}{1+s_{k j}^{(i)} p_{k j}^{(i)}(\mathbf{s})}+\gamma_{2} \frac{s_{k 0}^{(i)}}{A^{(i)}} \leq \lambda_{k}, \quad i=1, \cdots, N \\
\gamma_{2} \frac{\sqrt{s_{k 0}^{(i)} s_{j 0}^{(i)} p_{U_{j}}^{(i)}(\mathbf{s})}+s_{k 0}^{(i)} \sqrt{p_{U_{k}}^{(i)}(\mathbf{s})}}{A^{(i)} \sqrt{p_{U_{k}}^{(i)}(\mathbf{s})}} \leq \lambda_{k}, \quad i=1, \cdots, N
\end{gathered}
$$

where, each constraint is satisfied with equality, iff the respective power levels, $p_{k j}^{(i)}(\mathbf{s}), p_{U_{k}}^{(i)}(\mathbf{s}), k, j \in\{1,2\}$, are positive. These constraints are very similar to the optimality constraints in [7], but here there are $N$ times more constraints, which are all coupled across subchannels, through the same Lagrange multipliers $\lambda_{k}$ and $\gamma_{k}$; a feature which will prove useful for our iterative algorithm.

To obtain coherent combining gain, the cooperative powers $p_{U_{k}}^{(i)}(\mathbf{s})$ over a given subchannel should either be both positive, or both zero. When $p_{U_{k}}^{(i)}(\mathbf{s})>0,(21)$ is satisfied with equality for both TXs, and after some manipulation (20)-(21) yield

$$
\frac{s_{k j}^{(i)}}{1+s_{k j}^{(i)} p_{k j}^{(i)}(\mathbf{s})} \leq \frac{1}{\gamma_{1}} \frac{\lambda_{k}^{2} s_{j 0}^{(i)}}{\lambda_{j} s_{k 0}^{(i)}+\lambda_{k} s_{j 0}^{(i)}}
$$

$$
\frac{1-\gamma_{1}}{A^{(i)}}\left(s_{k 0}^{(i)}+\frac{\lambda_{k}}{\lambda_{j}} s_{j 0}^{(i)}\right)=\lambda_{k}
$$

The constraint (22) can be solved for $p_{k j}^{(i)}(\mathbf{s})$, to give

$$
p_{k j}^{(i)}(\mathbf{s})=\left(\frac{\gamma_{1}\left(\lambda_{j} s_{k 0}^{(i)}+\lambda_{k} s_{j 0}^{(i)}\right)}{\lambda_{k}^{2} s_{j 0}^{(i)}}-\frac{1}{s_{k j}^{(i)}}\right)^{+}
$$

This solution is quite interesting, as the power levels of the cooperative codewords on each subchannel should satisfy a single user water-filling type solution, and they depend only on the channel gains over that particular subchannel. The water level is determined by the direct link gains.

Similarly, solving (23) for $p_{U_{k}}^{(i)}(\mathbf{s})$, we obtain,

$$
\begin{aligned}
& p_{U_{k}}^{(i)}(\mathbf{s})=\left(\left(1-\gamma_{1}\right)\left(s_{k 0}^{(i)}+\left(\lambda_{k} / \lambda_{j}\right) s_{j 0}^{(i)}\right) / \lambda_{k}\right. \\
& \left.-\left(1+s_{k 0}^{(i)} p_{k j}^{(i)}(\mathbf{s})+s_{j 0}^{(i)} p_{j k}^{(i)}(\mathbf{s})\right)\right) s_{k 0}^{(i)} /\left(s_{k 0}^{(i)}+s_{j 0}^{(i)}\right)^{2}
\end{aligned}
$$

Note however that, this quantity is not guaranteed to be positive. In case it is not, this means that (23) is satisfied with strict inequality and the optimal solution for $p_{U_{k}}^{(i)}(\mathbf{s})$ should be set to 0 . But then, (24) is no longer valid, and instead, we should use, for $k=\{1,2\}$,

$$
\frac{\gamma_{1} s_{k j}^{(i)}}{1+s_{k j}^{(i)} p_{k j}^{(i)}(\mathbf{s})}+\frac{\left(1-\gamma_{1}\right) s_{k 0}^{(i)}}{1+s_{k 0}^{(i)} p_{k j}^{(i)}(\mathbf{s})+s_{j 0}^{(i)} p_{j k}^{(i)}(\mathbf{s})} \leq \lambda_{k}
$$

obtained from (20) by setting $p_{U_{k}}^{(i)}(\mathbf{s})=0$. This time, the power $p_{k j}^{(i)}(\mathbf{s})$ depends on $p_{j k}^{(i)}(\mathbf{s})$, and vice-versa: increasing one of the powers will decrease the other, should the constraints (26) be both satisfied with equality, and we now have a multiuser water-filling type solution. Note that, this is significantly different from the observations in [7], which assumed (22)-(23) were sufficient to cover all cases (for a scalar MAC).

When $p_{U_{k}}^{(i)}(\mathbf{s})=0, p_{k j}^{(i)}(\mathbf{s})$ can then be obtained by,

$$
\begin{aligned}
p_{k j}^{(i)}(\mathbf{s})= & \left(\frac{-b_{k}^{(i)}(\mathbf{s})+\sqrt{\Delta_{k}^{(i)}(\mathbf{s})}}{2 a_{k}^{(i)}(\mathbf{s})}\right)^{+} \\
a_{k}^{(i)}(\mathbf{s})= & \lambda_{k} s_{k 0}^{(i)} s_{k j}^{(i)}, \\
b_{k}^{(i)}(\mathbf{s})= & \lambda_{k}\left(s_{k 0}^{(i)}+s_{k j}^{(i)}+s_{k j}^{(i)} s_{j 0}^{(i)} p_{j k}^{(i)}(\mathbf{s})\right)-s_{k 0}^{(i)} s_{k j}^{(i)}, \\
c_{k}^{(i)}(\mathbf{s})= & \lambda_{k}\left(1+s_{j 0}^{(i)} p_{j k}^{(i)}(\mathbf{s})\right)-s_{k 0}^{(i)} \\
& -\gamma_{1}\left(s_{k j}^{(i)}+s_{j 0}^{(i)} s_{k j}^{(i)} p_{j k}^{(i)}(\mathbf{s})-s_{k 0}^{(i)}\right), \\
\Delta_{k}^{(i)}(\mathbf{s})= & b_{k}^{(i)}(\mathbf{s})^{2}-4 a_{k}^{(i)}(\mathbf{s}) c_{k}^{(i)}(\mathbf{s}),
\end{aligned}
$$

At this point, it should be clear that although (24) and (27) do not explicitly depend on $p_{U_{k}}^{(i)}(\mathbf{s})$, the decision regarding which of these equations should be used while computing $p_{k j}^{(i)}(\mathbf{s})$ does. Likewise, $p_{U_{k}}^{(i)}(\mathbf{s})$ are clearly functions of $p_{k j}^{(i)}(\mathbf{s})$, which makes equations (24), (25) and (27) coupled. Note however that, due to the convex nature of the problem, and the Cartesian nature of the constraints across TXs, it is possible to design an iterative algorithm, which performs updates on the powers of the TXs, one-user-at-a-time: given $p_{U_{1}}^{(i)}(\mathbf{s})$ and $p_{12}^{(i)}(\mathbf{s})$, we can compute $p_{U_{2}}^{(i)}(\mathbf{s})$ and $p_{21}^{(i)}(\mathbf{s})$, and using these new values for 
TX 2, we can re-iterate the powers of TX 1. Such an approach simplifies the seemingly difficult task of obtaining the optimal powers from the coupled equations, and provably converges to the optimal solution, as at the end of the iterations, the KKT conditions will be satisfied. The proposed algorithm is summarized as follows:

\section{Iterative Power Allocation Algorithm:}

- Fix the Lagrange multipliers $\lambda_{1}, \lambda_{2}$ and $\gamma_{1}$.

- For each subchannel, $i$ and each TX, $k$ :

- Calculate $p_{k j}^{(i)}(\mathbf{s})$ using (24) and $p_{U_{k}}^{(i)}(\mathbf{s})$ using (25) assuming $p_{U_{k}}^{(i)}(\mathbf{s})>0$

- If $p_{U_{k}}^{(i)}(\mathbf{s})<0$, then for those $\mathbf{s}$, set $p_{U_{k}}^{(i)}(\mathbf{s})=0$ and re-calculate $p_{k j}^{(i)}(\mathbf{s})$ using (27) and $p_{U_{k}}^{(i)}(\mathbf{s})$ using (25)

- Iterate this procedure across TXs, until all KKT conditions are satisfied for given $\lambda_{1}, \lambda_{2}$ and $\gamma_{1}$.

- Iteratively update $\lambda_{1}, \lambda_{2}, \gamma_{1}$, until average power constraints (17)-(18) and rate constraints (15)-(16) satisfied.

Perhaps the most important feature of this algorithm is that, regardless of the number of subchannels used, we only need to solve for three Lagrange multipliers, which relate the powers allocated to the subchannels, to obtain the optimum PA. This reduces the complexity of the algorithm dramatically, and makes it scalable, compared to the subgradient algorithm. As a result, the convergence is much faster.

\section{Simulation Results}

In this section, we first simulate the projected subgradient algorithm in order to obtain the optimal power allocation, and the resulting achievable rate region for a simple case with only three subchannels. The achievable rate region for the ISCE strategy is obtained by running this algorithm for varying priorities $\mu_{k}$, and then by taking a convex hull over the resulting power optimized regions. In Figure 3, we compare the achievable rate region for power controlled cooperative OFDMA with those for several enconding strategies without power control, from [1]. We assume that, for the channel non-adaptive protocols, the TXs are still able to allocate their total power across subchannels and codewords. The total power of each TX and the noise variances are set to one. The fading coefficients are chosen from independent Rayleigh distributions, the means of which are shown in Figure 3. We observe that, when the powers are chosen jointly optimally with ISCE, there is a major improvement in achievable rates. This unusually high gain from power control can be attributed to our ability to take advantage of the additional diversity created by OFDMA: power allocation not only allows us to use the subchannels at time varying instantaneous rates based on the channel qualities, but also to use them adaptively for varying purposes, i.e., cooperation, common message generation or direct transmission.

The simulations of the iterative algorithm proposed in Section IV-B to maximize the sum rate are carried out for an independent uniform fading scenario, to guarantee operation in case 1. Figures 4(a)-4(c) and 5(a)-5(c) demonstrate the powers allocated to subchannel 1 , as functions of the inter-TX link gains, when the direct link gains are fixed to two different sets specified on the figures. Powers $p_{U_{2}}^{(1)}$ are not shown, to save

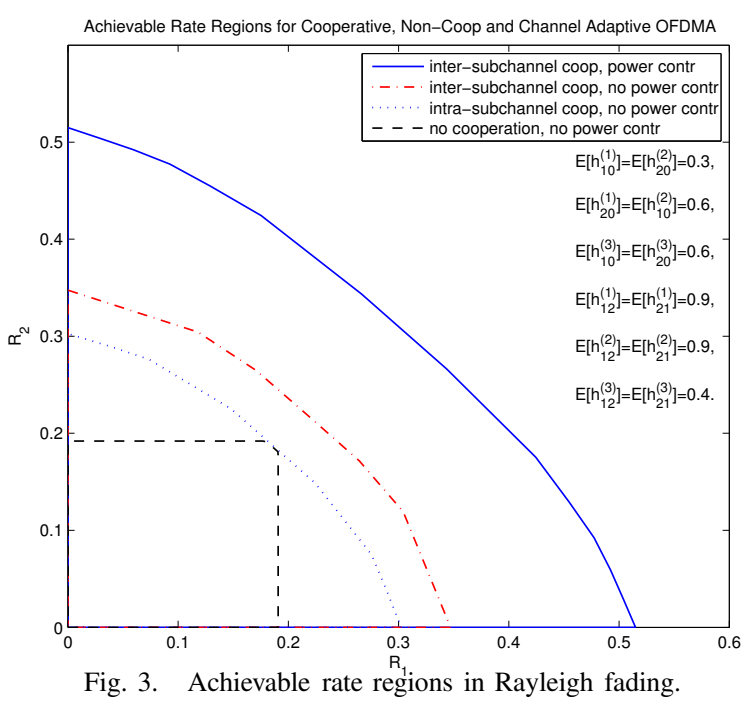

space, as they are identical to $p_{U_{1}}^{(1)}$ due to the symmmetry in fading. An important observation is that, although we make no prior assumptions on subchannel allocation to TXs/codewords, the optimal powers sometimes dictate exlusive use of some subchannels for dedicated tasks. For an example, see Figures 5(a)-5(c): when the inter-TX links are both strong, the TXs exchange information using simultaneous waterfilling. When only $s_{21}^{(1)}$ is strong, only TX 2 uses the subchannel. When both inter-TX links are weak, the TXs use the subchannel solely to convey common information to the RX, by using only $p_{U_{1}}^{(1)}$ and $p_{U_{2}}^{(1)}$. The resulting power distributions show that the KKT conditions are indeed satisfied at the fixed point of our iterative algorithm, verifying convergence.

In Figures 6(a)-6(c), we plot the power distributions obtained using the subgradient algorithm instead, for the same setting as in Figures 5(a)-5(c). The subgradient algorithm is terminated after 10000 iterations. It is observed that while the powers $p_{12}^{(1)}(\mathbf{s})$ and $p_{21}^{(1)}(\mathbf{s})$ seem to have nearly converged to the optimal values shown in Figures 5(a)-5(c), the cooperative power $p_{U_{1}}^{(1)}(\mathbf{s})$ has still not fully converged, though it is close to its optimal distribution. The sum rate obtained by the subgradient algorithm is 0.2239 bits/transmision, whereas the faster iterative algorithm terminates at the optimum value of 0.2241 bits/transmission.

\section{CONCLUSION}

We obtained the optimum power allocation policies for a cooperative OFDMA channel that employs ISCE. We developed a subgradient algorithm which converges to the optimum power allocation policies that achieve the maximum rate region, and a more efficient iterative algorithm which maximizes the sum rate. We demonstrated that the optimal power allocation may also serve as a guideline for subchannel assignment to the TXs' cooperative codewords, and that power allocation for cooperative OFDMA provides significant rate improvements, due to its ability to exploit the diversity provided by OFDMA. The resulting rate regions serve as benchmarks for several practical single cell and multicell systems, as the techniques developed are readily extendible to multiuser scenarios, by running the proposed algorithms for multiple pairs of users operating in parallel over orthogonal subchannels. 


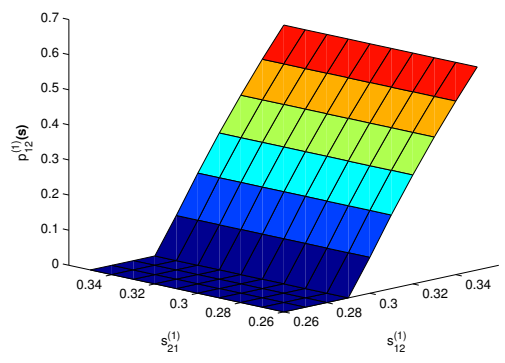

(a) Power level, $p_{12}^{(1)}$

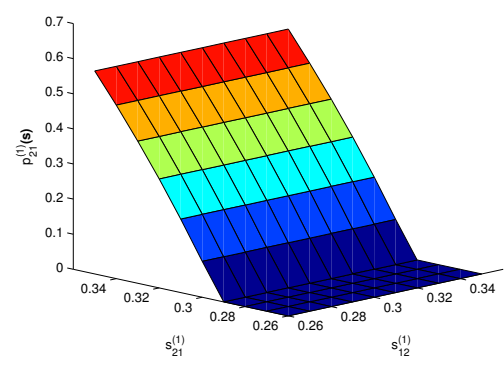

(b) Power level, $p_{21}^{(1)}$

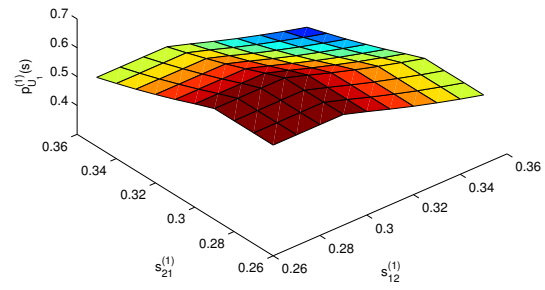

(c) Power level, $p_{U 1}^{(1)}=p_{U 2}^{(1)}$

Fig. 4. Optimal power allocation when $s_{10}^{(1)}$ and $s_{20}^{(1)}$ are maximum (i.e. $\left.s_{10}^{(1)}=s_{20}^{(1)}=0.25\right)$, fixed and always less than $s_{12}^{(1)}$ and $s_{21}^{(1)} \cdot p_{U_{k}}^{(1)}$ are always positive, to take advantage of strong direct links. $p_{k j}^{(1)}$ obey single user waterfilling, as expected.

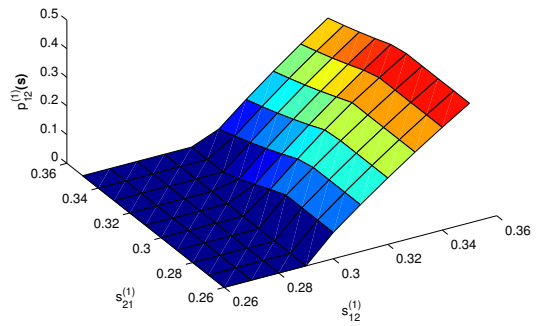

(a) Power level, $p_{12}^{(1)}$

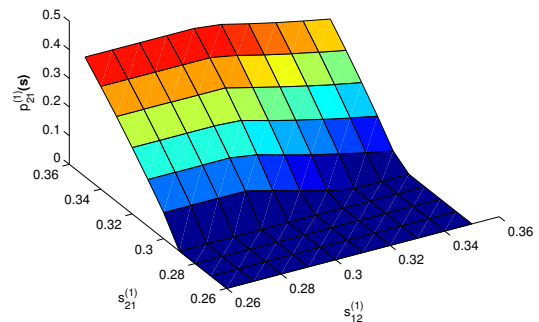

(b) Power level, $p_{21}^{(1)}$

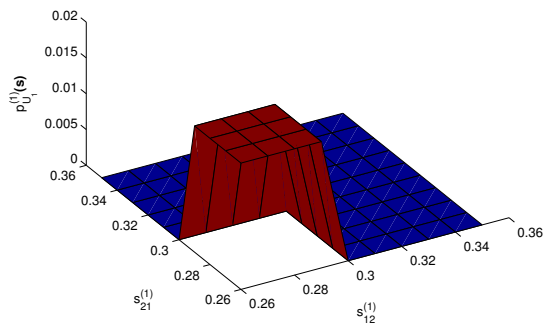

(c) Power level, $p_{U 1}^{(1)}=p_{U 2}^{(1)}$

Fig. 5. Optimal power allocation when $s_{10}^{(1)}=s_{20}^{(1)}=0.15$, fixed and always less than $s_{12}^{(1)}$ and $s_{21}^{(1)}$. When $p_{U_{k}}^{(1)}$ is positive, $p_{k j}^{(1)}$ obey single user waterfilling. As the inter-TX links get stronger, it becomes more profitable to create common information, $p_{U_{k}}^{(1)}$ become 0 , and the TXs perform simultaneous waterfilling.

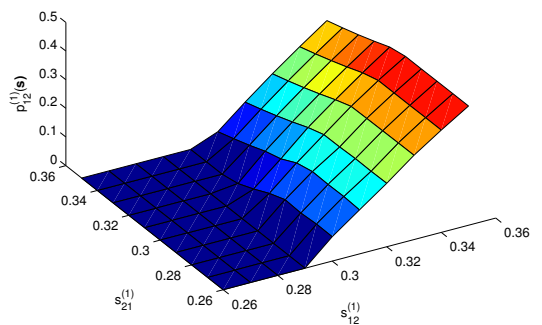

(a) Power level, $p_{12}^{(1)}$

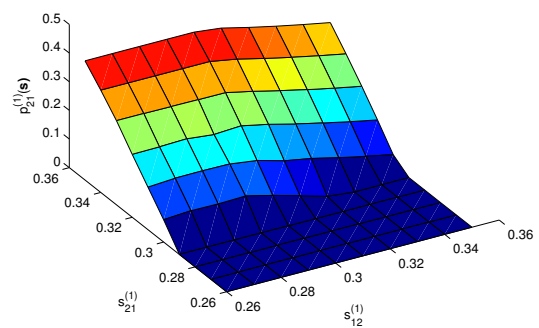

(b) Power level, $p_{21}^{(1)}$

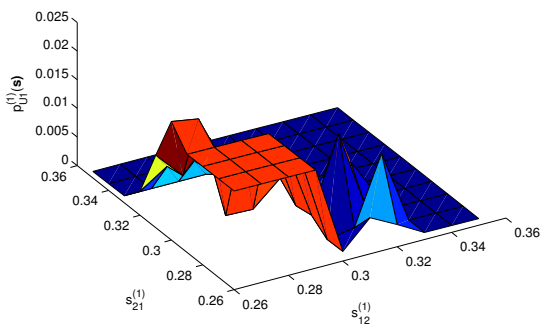

(c) Power level, $p_{U 1}^{(1)}$

Fig. 6. Power allocation obained after 10000 iterations of the subgradient algorithm, when $s_{10}^{(1)}=s_{20}^{(1)}=0.15$, fixed and always less than $s_{12}^{(1)}$ and $s_{21}^{(1)}$. The algorithm has not yet converged to the optimum value, despite a much longer running time compared to the iterative algorithm. Achievable rates are within $0.2 \%$ of the optimum value.

\section{REFERENCES}

[1] S. Bakım and O. Kaya. "Achievable Rates for Two User Cooperative OFDMA." In Proc., IEEE Globecom, Miami, FL, Dec. 2010.

[2] K. Kim, Y. Han and S.-L. Kim. "Joint Subcarrier and Power Allocation in Uplink OFDMA Systems.” IEEE Commun. Lett., 9(6): 526-528, Jun. 2005.

[3] C. Ng and C. Sung. "Low Complexity Subcarrier and Power Allocation for Utility Maximization in Uplink OFDMA Systems." IEEE Trans. Wireless Commun., 7(5): 1667-1675, May 2008.

[4] L. Gao and S. Cui. "Efficient Subcarrier, Power and Rate Allocation with Fairness Consideration for OFDMA Uplink." IEEE Trans. Wireless Commun., 7(5): 1507-1511, May 2008.

[5] F. M. J. Willems, E. C. van der Meulen and J. P. M. Schalkwijk. "An Achievable Rate Region for the Multiple Access Channel with Generalized Feedback." In Proc. Allerton Conference, Monticello, IL, Oct. 1983

[6] A. Sendonaris, E. Erkip and B. Aazhang. "User Cooperation Diversity - Part I: System Description.” IEEE Trans. Commun., 51(11): 19271938, Nov. 2003.

[7] O. Kaya and S. Ulukus. "Power Control for Fading Cooperative
Multiple Access Channels." IEEE Trans. Wireless Commun., 6(8): 2915-2923, Aug. 2007.

[8] L. Weng and R. D. Murch. "Cooperation Strategies and Resource Allocations in Multiuser OFDMA Systems." IEEE Trans. Veh. Technol., 58(5): 2331-2342, Jun. 2009.

[9] W. Shim, Y. Han and S. Kim. "Fairness-Aware Resource Allocation in a Cooperative OFDMA Uplink System." IEEE Trans. Veh. Technol., 59(2): 932-939, Feb. 2010.

[10] Z. Han, T. Himsoon, W. P. Siriwongpairat and K. J. R. Liu. "Resource Allocation for Multiuser Cooperative OFDM Networks:Who Helps Whom and How to Cooperate." IEEE Trans. Veh. Technol., 58(6), 2378-2391, Jun. 2009.

[11] A. J. Goldsmith and P. P. Varaiya. "Capacity of Fading Channels with Channel Side Information.” IEEE Trans. Inf. Theory, 43(6):1986-1992, Nov. 1997.

[12] D. Tse and S. Hanly. "Multiaccess Fading Channels - Part I: Polymatroid Structure, Optimal Resource Allocation and Throughput Capacities.” IEEE Trans. Inf. Theory, 44(7): 2796-2815, Nov. 1998.

[13] N. Z. Shor. Minimization Methods for Non-Differentiable Functions. New York: Springer-Verlag, 1979. 